\title{
Diseño y construcción de microaerogenerador "AR-SF-WP-500" para la molienda de mineral a escala de laboratorio
}

Alfonso A. Romero ${ }^{1}$

Silvana L. Flores ${ }^{2}$

Werner W. Pacheco ${ }^{3}$

\begin{abstract}
RESUMEN
El micro-aerogenerador, denominado AR-SFWP-500, posee un dínamo que genera una potencia de 500 Watts, el cual se caracteriza por tener en su estructura interna, imanes permanentes (GIP) para trabajar con microaerogeneradores, como para emplearlos en zonas rurales, donde hay carencia de energía eléctrica. El propósito del artículo, consiste en explicar el proceso del diseño y la construcción del Microaerogenerador AR-SF-WP-500, cuyo principio de funcionamiento se basa en convertir la energía eólica en energía eléctrica, de tal manera que este tipo de energía se use para mover el molino de mineral de escala de laboratorio, el cual emplea un motor eléctrico de baja potencia.
\end{abstract}

Palabras clave: Energía eólica, microaerogenerador, molino de mineral.

DESIGN AND CONSTRUCTION OF THE SMALL WIND GENERATOR CALLED "AR-SF-500" FOR THE ORE MILLING TO A LABORATORY SCALE ABSTRACT

The small wind generator called "AR-SFWP-500" has a dynamo that generated a potency of 500 watts, which characterize for having in its internal structure, the called "permanent magnet" for working with small wind generators, such as employing in rural zones, where there is a lack of electric energy. In this sense, the purpose of the paper, treats explaining the process of design and construction of the Small Wind Generator AR-SFWP-500, whose principle of functioning is based in transform the wind energy in electric energy, in such manner, this kind of energy has been used for moving the ore milling at laboratory scale, which uses an electric motor of a lower potency.

Key words: Wind energy, wind generator, ore milling.

\section{INTRODUCCIÓN}

El presente trabajo se basó en un proceso de diseño y fabricación de un microaerogenerador, con la finalidad de poder mover un molino de mineral a escala de laboratorio.

El empleo de un micro-aerogenerador para convertir la energía del viento o eólica en energía eléctrica será usada en el funcionamiento de un molino de mineral, todo lo cual depende de la potencia del dínamo para un motor eléctrico de baja potencia.

La parte experimental del diseño y la construcción del microaerogenerador y el molino de mineral, se realizó en el taller de mecánica de la empresa Tecnologías Múltiples S.A.C.

Las pruebas de pilotaje del funcionamiento del molino de mineral impulsado por el micro-aerogenerador, se realizo en el Laboratorio de Investigaciones Multidisciplinarias de la Escuela de Minas.

El propósito del estudio fue demostrar que existe una generación de movimiento del molino de mineral, considerando la potencia de energía eléctrica proporcionada por el dínamo del micro-aerogenerador, considerando la eficiencia de aprovechamiento de la energía eólica, que se da por el componente radial, vale decir que es la fuerza que hace girar el molino de viento se convierte en movimiento del rotor, cuyo componente corresponde al $35 \%$ de la presión dinámica del aire; en los molinos de viento más desarrollados, conociendo que el movimiento del rotor genera un cierto torque que multiplicado por la velocidad de giro da la potencia real del molino de viento.

\section{FUNDAMENTO TEÓRICO}

\subsection{MICROAEROGENERADOR [2]}

El aerogenerador de tipo eje horizontal, es considerado el más eficiente y más usado en la actualidad, puesto que extrae la energía del viento utilizando una tecnología que se asemeja a la de los aviones o helicópteros.

Los microaerogeneradores o turbinas de viento tienen varios componentes importantes que cumplen una función determinada en el principio de conversión de energía eólica en energía eléctrica.

\footnotetext{
Docente de la Escuela de Ingeniería de Minas de la Universidad Nacional Mayor de San Marcos. E-mail: aromerob@unmsm.edu.pe

2 Docente de la Escuela de la Facultad de Ingeniería Electrónica y Eléctrica.

E-mail: sfloresc@unmsm.edu.pe

3 Docente de la Escuela de Ingeniería de Minas.

E-mail: wpachecol@unmsm.edu.pe
} 
A continuación, se señalarán las funciones principales de cada uno de los componentes del microaerogenerador.

\subsubsection{ROTOR [2]}

Este dispositivo conforma la parte externa del microaerogenerador. Se fabrica considerando el empleo de una plancha de fierro de $1 / 4 \mathrm{de} \mathrm{mm}$ de espesor.

El rotor consiste básicamente en un disco de $11 \mathrm{~cm}$ de diámetro, en cuya área se sueldan tres platinas, cada una de las cuales posee $67 \mathrm{~cm}$. de longitud y $1 / 8$ de pulgadas de espesor y de $1 \mathrm{~cm}$ de ancho.

Es en ese sentido, que se puede afirmar que el rotor poseerá tres platinas, en cada una de las cuales se adherirán las palas del microaerogenerador.

El diseño del rotor permite que en la parte central vaya alojado un tope, que consiste en una arandela gruesa de acero soldada al disco del rotor, de $1 / 4$ de pulgada de diámetro y de $1 / 8$ de pulgada de espesor. Este tope permite al rotor poder apoyarse y girar sobre el eje dinámico montado sobre las chumaceras que forman parte de la tornamesa.

De esta manera, se afirma que el rotor incluye tres palas, puesto que la función de las palas será capturar el viento y transmitir la potencia hacia la tornamesa que está acoplada al eje de baja velocidad del microaerogenerador.

De esta manera, el rotor convierte la fuerza del viento en energía rotatoria del eje, una caja de engranajes aumenta la velocidad y un generador transforma la energía del eje en energía eléctrica.

Los modelos que se instalan en la actualidad son, por lo general, tripala, de paso variable (este sistema permite una producción óptima con vientos bajos y una reducción de cargas con vientos altos), de alta calidad en el suministro eléctrico y bajo mantenimiento.

Preparadas para optimizar los recursos eólicos de un emplazamiento determinado, su vida útil de estas máquinas es, como mínimo, de 20 a 25 años, aunque resulta posible que en algunos emplazamientos puedan ser sustituidos por otros equipos de mayor eficacia antes de finalizar su vida útil.

\subsubsection{DÍNAMO [1]}

Es un generador eléctrico de corriente alterna trifásica (AC) de bajo voltaje.

La corriente se transforma a una corriente continua (DC) mediante diodos rectificadores, con la finalidad de que la energía pueda ser almacenada en baterías.

\subsubsection{TORRE [1]}

Soporta la cola y el rotor. Puede ser tubular o de celosía (estas últimas, apenas se emplean en los grandes aerogeneradores debido a que generan un mayor impacto visual).

\subsection{POTENCIA GENERADA [2]}

La potencia suministrada por un aerogenerador depende de la superficie barrida por la hélice $y$, por tanto, de la longitud de las palas y es independiente del número de palas.

La energía que origina el viento es energía cinética, es decir, está causada por la altura del aire en movimiento:

$$
E_{c}=1 / 2 m V^{2}
$$

Donde:

M: es la masa del aire en $\mathrm{Kg}$, y V: es la velocidad instantánea del viento $(\mathrm{m} / \mathrm{seg})$.

La masa de esta cantidad de aire es:

$$
\mathrm{m}=\Phi . \mathrm{V}
$$

Donde:

$\Phi$ : Es la densidad de aire, $1.25 \mathrm{~kg} / \mathrm{m}^{3}$, y V, el volumen del cilindro barrido.

El volumen del cilindro es:

$$
V=A . L
$$

Donde:

\section{A: Es la superficie barrida}

L: La longitud del cilindro, que resulta un espacio $y$, por tanto, es igual a una velocidad (la del viento para un tiempo (en segundos).

$$
L=v . t
$$

Con todo ello, se determina que la energía es igual a:

$$
\begin{gathered}
E_{c}=1 / 2 \Phi \cdot V \cdot v^{2}=1 / 2 \Phi \cdot A \cdot L \cdot v^{2}=1 / 2 \Phi . \\
\text { A.v.t. } v^{2}=1 / 2 \Phi \cdot A \cdot v^{2} \cdot t
\end{gathered}
$$

Por tanto, la potencia teórica del viento será:

$$
P=E c / t=1 / 2 A \cdot \Phi \cdot v^{2}
$$

Si el área barrida transversalmente por el viento es circular, como ocurre en todas las máquinas eólicas de eje horizontal,

$$
A=p . D^{2} / 4
$$

Siendo D, el diámetro; por lo que la potencia disponible será:

$$
P=p / 8 . \Phi \cdot D^{2} \cdot v^{3}
$$


La energía almacenada en el viento es muy elevada, pero es difícil extraer al $100 \%$. Albert Betz demostró en 1919 que para extraer la energía del viento se debe reducir su velocidad.

Teóricamente, para captar el cien por cien de la energía que transporta en forma de energía cinética, se debería detener completamente el aire en movimiento. Pero si se detuviera al aire totalmente al estar parado, impediría la entrada de más aire el rotor de la turbina; y de esta manera no se podría captar más energía.

Para evitar esto, se debe permitir que el viento escape con cierta velocidad, reduciendo el rendimiento de producción energética, pero permitiendo que más viento pueda alcanzar el microaerogenerador.

\section{METODOLOGÍA}

En el presente paper, se definirán los principales componentes del microaerogenerador, el procedimiento para la fabricación de moldes, plantillas, los gráficos con las dimensiones del rotor; posteriormente se presentarán los materiales y herramientas básicas el diseño y la construcción del microaerogenerador empleadas y por último, se explicará el procedimiento de fabricación del microaerogenerador de manera integral.

Todo el proceso de construcción del microaerogenerador se realizará considerando el estudio de los parámetros de diseño.

Primero, se estudiarán los parámetros de diseño a tener en consideración cuando se construye un microaerogenerador, posteriormente se procederá a la construcción propiamente dicha.

\subsection{PARÁMETROS DE DISEÑO DEL MICROAE- ROGENERADOR AR-SF-WP-500 [2]}

\subsubsection{Velocidades del viento}

Los microaerogeneradores que se emplean para producir electricidad tienen como parámetro importante, la fuerza del viento. Siendo el inconveniente principal que tiene este tipo de energía, su discontinuidad, con momentos de calma, variación de su velocidad y cambios en la dirección e intensidad.

Cabe resaltar que para el aprovechamiento de la energía eólica y su transformación en energía eléctrica se necesitan las siguientes velocidades del viento.
Tabla N..$^{\circ}$ 1. Velocidades del viento necesarias para transformación del viento en energía eólica.

\begin{tabular}{|c|c|c|}
\hline Velocidad del viento & $\mathrm{Km} / \mathrm{h}$ & $\mathrm{m} / \mathrm{s}$ \\
\hline $\begin{array}{c}\text { Velocidad mínima } \\
\text { para el inicio de giro }\end{array}$ & 19 & 5 \\
\hline $\begin{array}{c}\text { Velocidad que } \\
\text { proporciona máximo } \\
\text { rendimiento }\end{array}$ & 40 a 48 & 11 a 13 \\
\hline $\begin{array}{c}\text { Velocidad máxima de } \\
\text { funcionamiento }\end{array}$ & 100 & 28 \\
\hline
\end{tabular}

Fuente. Roldan José, Fuentes de Energía, Cengage Learning Paraninfo S.A., Madrid, España, 2008.

\subsubsection{Eje horizontal con tres palas [1]}

El modelo elegido para la construcción del aerogenerador ha sido el de eje horizontal con tres palas, puesto que los microaerogeneradores de este tipo extraen la energía del viento utilizando una tecnología que se asemeja a la de los aviones o helicópteros.

Cabe resaltar que el secreto del rendimiento de las máquinas de eje horizontal es que no solo trabajan con el empuje del viento, sino en combinación con la fuerza de sustentación.

Una hélice bien diseñada busca la combinación de ángulos y formas para evitar turbulencia y sacar el máximo rendimiento de una hélice, hay que darle la forma adecuada, curvatura a lo largo de su longitud e inclinación. Este conjunto conlleva un intenso y complejo proceso de cálculos, ensayos y experimentación aerodinámicos.

Es en ese sentido, que para este tipo de máquinas, la potencia nominal teórica en Vatios viene dada por la fórmula:

$$
P=0.2^{*} d^{2 *} v^{3}
$$

Donde:

$\mathrm{P}=$ potencia en $\mathrm{W}$

$\mathrm{D}=$ diámetro del rotor en $\mathrm{m}$

$\mathrm{V}=$ velocidad del viento en $\mathrm{m} / \mathrm{seg}$

\subsubsection{Revoluciones de aerogenerador [2]}

El número de revoluciones máximo del rotor se calcula mediante la siguiente expresión, si se sobrepasara esta cifra puede ser peligroso para el sistema:

$N^{\circ}$ R.P.M. máx. $=2000 /$ diámetro $(\mathrm{m})$ 
El diámetro del rotor es de $11 \mathrm{~cm}$

$$
\text { No R.P.M. máx. }=2000 /(0.11)=18181.82
$$

Los aerogeneradores o turbinas de viento tienen varios componentes. El rotor convierte la fuerza del viento en energía rotatoria del eje, una caja de engranajes aumenta la velocidad y un generador transforma la energía del eje en energía eléctrica.

\subsection{CONSTRUCCIÓN DEL MICROAEROGENE- RADOR [2]}

Para realizar las pruebas experimentales del presente estudio, se requirió las instalaciones del taller de mecánica de la empresa "Tecnologías Múltiples" y las instalaciones del Laboratorio de Investigaciones Multidisciplinarias de la Escuela de Ingeniería de Minas de la UNMSM. Ver Figuras No. 3 y 4

Para el desarrollo del estudio, se tuvo en cuenta los siguientes materiales y herramientas.

\subsubsection{Material y herramientas}

\subsubsection{Materiales para la construcción}

Los materiales necesarios utilizados para la fabricación de un microaerogenerador son:

- Plancha de acero de $1 / 4$ de pulgada de espesor.

- Perfiles de 1 X 1/8"

- Eje trefilado de $53 \mathrm{~cm}$ de largo y 1 pulgada de diámetro, y punta roscada de $2.5 \mathrm{~cm}$ en un extremo.

- Arandela gruesa de 1/4" de diámetro y 1/8" de espesor.

- Chumaceras de tipo pie de una pulgada de diámetro y pernos de 3/8" para formar la base horizontal de la tornamesa.

- Plancha de tetrapack de aluminio reciclado.

- Dínamo.

- Una polea de aluminio de $13 \mathrm{~cm}$ de diámetro y 1 $\mathrm{cm}$. de diámetro central.

- Tuercas de 1/4" X 1", 3/8" X 1" con arandelas y porta-arandelas.

- Soldadura.

- Platina de $8 \mathrm{~cm}$ de longitud X $2 \mathrm{~cm}$ de ancho $X$ $20 \mathrm{~mm}$ de espesor.

- Platina de $6 \mathrm{~cm}$ de longitud X $3 \mathrm{~cm}$ de ancho $X$ $20 \mathrm{~mm}$ de espesor.

- Platina 3/16" de espesor X 1“de largo.
- Ángulos de 1 X 1/8" de espesor.

- Ángulos de 9 X 3/16" de espesor.

- 2 ángulos de $28 \mathrm{~cm}$ de largo, que irán soldados para formar la base horizontal de la tornamesa.

- 2 ángulos de $14 \mathrm{~cm}$ de ancho, que irán soldados para formar la base horizontal de la tornamesa.

- Tubo de fierro de $15 \mathrm{~cm}$ de largo, $6 \mathrm{~cm}$ de diámetro externo y $5 \mathrm{~cm}$ de diámetro interno para la base que se suelda en el marco del ángulo de la punta de la torre del microaerogenerador.

- Tubo de fierro de $13 \mathrm{~cm}$ de largo, $6 \mathrm{~cm}$ de diámetro externo y $5 \mathrm{~cm}$. de diámetro interno, que sirve para la parte superior que sujeta a la tornamesa.

- Tubo de fierro de $66 \mathrm{~cm}$ de largo, $4.6 \mathrm{~cm}$ de diámetro externo y $4.1 \mathrm{~cm}$ de diámetro interno, que sirve para soportar a la tornamesa.

- Tubo de fierro de $30 \mathrm{~cm}$ de longitud, $4.6 \mathrm{~cm}$ de diámetro externo y $4 \mathrm{~cm}$ de diámetro interno, que sirve para soportar a la tornamesa de manera horizontal.

- Tubo de fierro de $22 \mathrm{~cm}$ de longitud, $4 \mathrm{~cm}$ de diámetro externo y $3.1 \mathrm{~cm}$ de diámetro interno, que sirve para soportar a la tornamesa de manera vertical.

- Tubo de fierro en el cual se sueldan 4 platinas, cada platina posee $3 \mathrm{~cm}$ de longitud y $1.5 \mathrm{~cm}$ de ancho.

- O Ring de $14.8 \mathrm{~cm}$ de diámetro.

- Inversor de 12 VDC a 220 VAC.

- 2 baterías de 15 placas.

- Terminal de batería.

- Cable mellizo N. ${ }^{\circ} 14$ tipo automotriz.

\subsubsection{HERRAMIENTAS PARA LA CONSTRUC- CIÓN}

Lista de herramientas básicas necesarias para la construcción del microaerogenerador:

- Máquina de soldar.

- Dobladora.

- Taladro de pie.

- Taladro de mano.

- Brocas N. ${ }^{0} 1 / 4 ", 3 / 8 "$.

- Desarmadores.

- Cincel. 


\subsubsection{DISEÑO DEL MICROAEROGENERADOR}

La turbina eólica es el componente del aerogenerador compuesto por tres palas que se encuentran expuestas al viento. En otras palabras, la turbina eólica se refiere básicamente al rotor eólico o conjunto de paletas que giran sobre un eje perpendicular al plano formado por ellas.

Para la fabricación de las paletas se requirió de un molde patrón, el cual debe ser construido con los datos obtenidos de parámetros como velocidad de viento, velocidad de giro, etc.

Es en ese sentido que se presentan los datos para construir las secciones para la construcción del molde, así como los materiales requeridos y sus características requeridas; asimismo, se presentará el procedimiento de fabricación de las paletas, la tornamesa, y los accesorios tales como: el porta dínamo, la cola y la portacola.

\section{FABRICACIÓN DE LA PLANTILLA DE LAS PA- LAS.}

Las palas van acopladas en forma equidistante alrededor del microaerogenerador, por medio de pernos y tuercas. Para realizar los agujeros de estos pernos, se requiere preparar una plantilla que permita tener una mejor precisión en la perforación. Esta misma plantilla también deberá ser utilizada para hacer los agujeros en la base de las palas, lo cual asegurará que todos los agujeros coincidan de la manera más exacta posible.

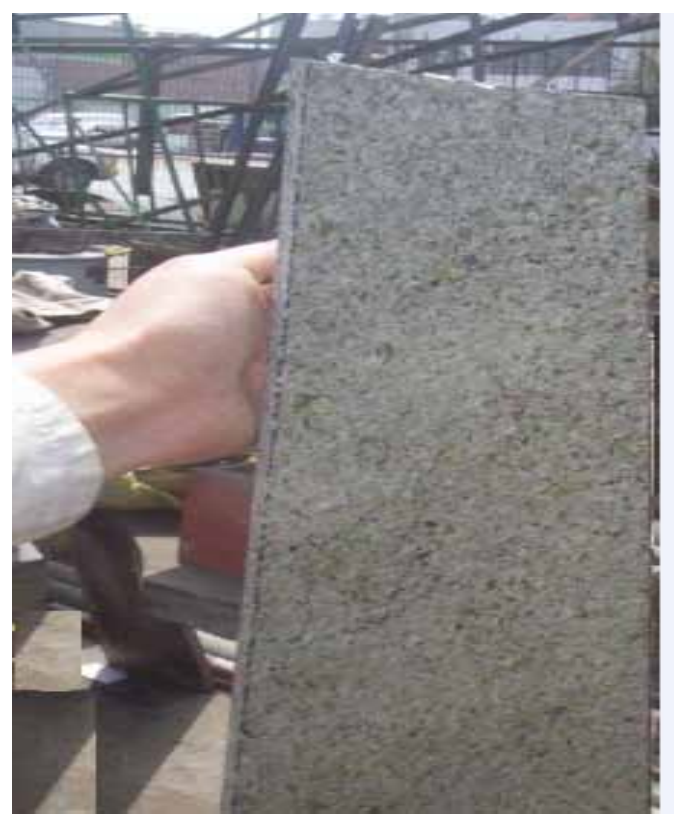

Figura $\mathbf{N}^{\circ}{ }^{1}$. Trazado de dimensiones de la plancha de tetrapack de aluminio.
Para la realización de esta plantilla, se requieren de los siguientes materiales:

Cartón grueso de tetrapack de aluminio reciclado, regla, lápiz, y tijera.

Los pasos empleados para la construcción de la plantilla de las palas son:

\section{PASO N. ${ }^{\circ} 1$}

- Trazado de la plancha original de tetrapack de aluminio de 28 pulgadas de ancho y 28 pulgadas de largo, una plancha de forma de un trapecio de base superior de 5 pulgadas y una base inferior de 9 pulgadas, de tal manera que se obtenga palas de forma trapezoidal. Ver figuras $\mathrm{N} .^{\circ}$ 1 y 2.

\section{PASO N. ${ }^{\circ} 2$}

- Doblado de los moldes trapezoidales para ser empleadas como palas del microaerogenerador, de tal manera que se logre dar un ángulo de doblez de 15 grados a las palas, puesto que el aumento de ángulo produce que el rotor comience a girar a una velocidad del viento reducida y además, evita que se embale de revoluciones a velocidades del viento excesivas, ya que al estar el ángulo algo sobrepasado produce un elevado de rozamiento con el aire a altas velocidades de giro. Ver figura $\mathrm{N}^{\circ} 3$.

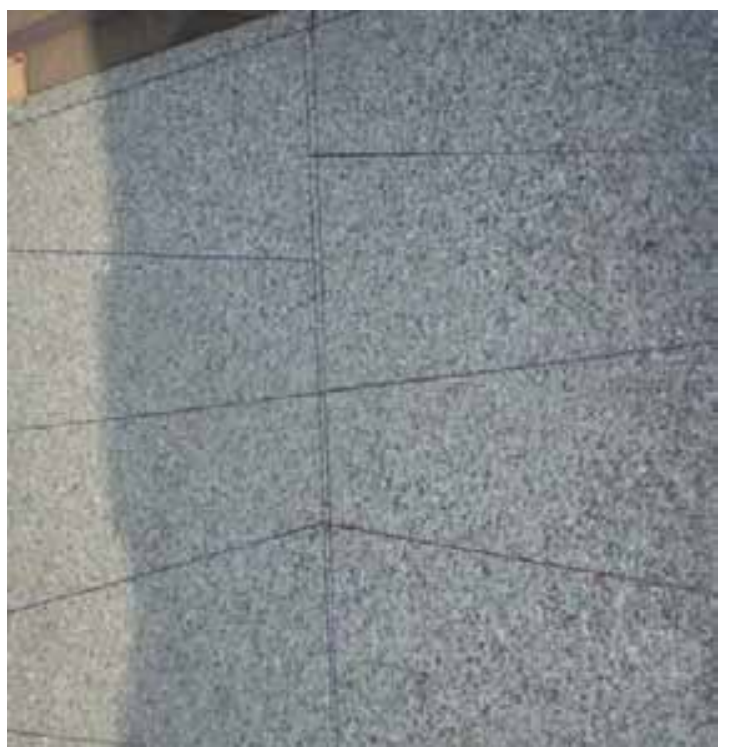

Figura $\mathbf{N}^{\circ}{ }^{2}$ 2. Corte de la plancha de tetrapack de aluminio. 


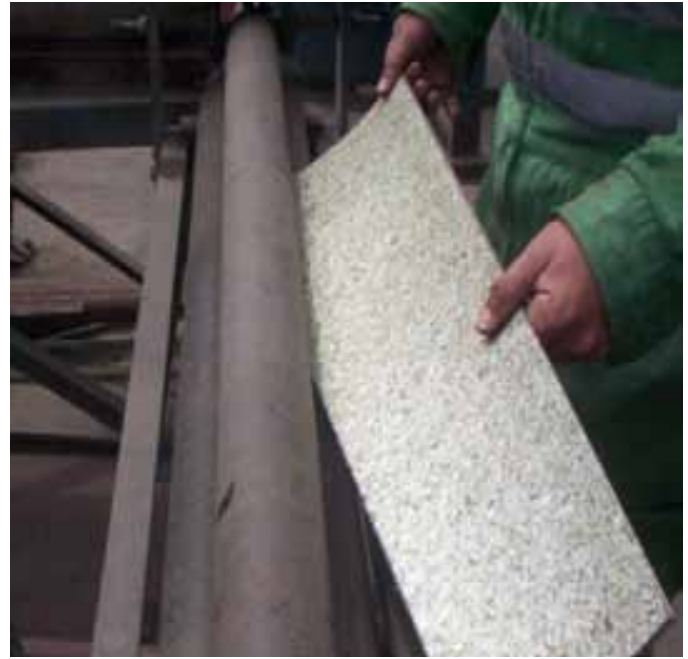

Figura $\mathbf{N}^{\circ}{ }^{3}$. Doblado de la plancha de tetrapack de aluminio.

\subsection{PROCESO DE FABRICACIÓN DEL ROTOR}

Antes de realizar la fabricación del rotor del aerogenerador, es necesario conocer cuáles son sus características. A continuación mencionaremos las características más sobresalientes:

- El rotor se compone de un disco con un eje central, sobre el cual se insertan tres paletas fabricadas de platinas, cada una de las cuales son equidistantes respecto al centro del disco del rotor. Estas palas posteriormente son protegidas con una pintura anticorrosiva denominada "Zinc Cromato".

- El rotor posee una velocidad máxima de 18,182 rpm.

- El rotor tiene un diámetro nominal de $11 \mathrm{~cm}$.

- Se ensambla de manera directa con el eje horizontal que está inserto en la tornamesa. Ver figura N. ${ }^{\circ} 4$.

\subsection{PROCESO DE INSTALACIÓN DEL MI- CROAEROGENERADOR AR-SF-WP-500}

El proceso de instalación del microaerogenerador AR-SF-WP-500, consiste en:

1) Armar el aerogenerador completo con el asta (torre) inclinada sobre un caballete en un ángulo de $20^{\circ}$ con respecto al eje horizontal, previo a ello, se debe ubicar el caballete a 3/5 partes de largo total, con el lado más largo hacia la base y ubicar la base cuadrada hacia los pernos de anclaje para facilitar su instalación.

2) Atar 4 cabos de 1/2" de diámetro mínimo a cuatro posiciones en el extremo superior de la torre, a $90^{\circ}$ entre cabo y cabo.

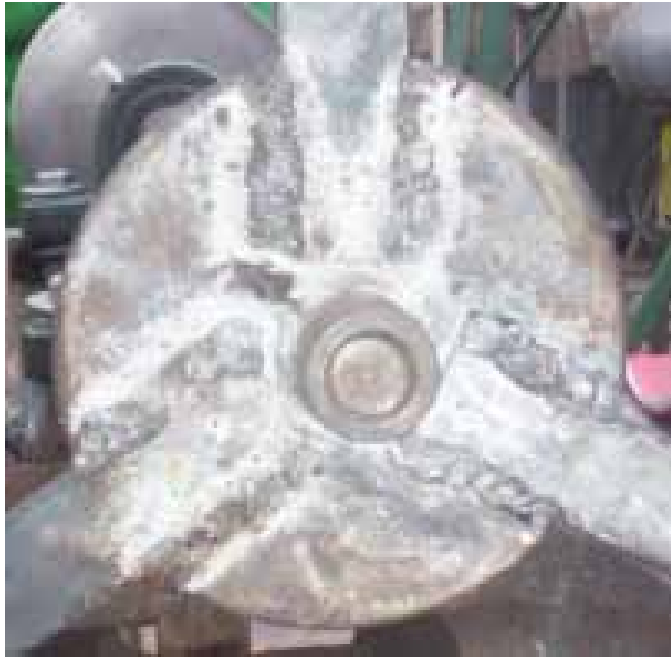

Figura $N^{\circ}{ }^{4}$. Rotor inserto en tornamesa.

3) Izar el equipo utilizando el cabo posterior como tractor y los otros tres como templadores para mantener la estabilidad del equipo en la izada.

4) Una vez izada la torre, asegurar la base a los pernos de anclaje, instalar los templadores y regular la perpendicularidad con respecto al pico.

5) Realizar las pruebas aerodinámicas y eléctricas antes de conectar las baterías.

6) Las pruebas resultaron satisfactorias al conectar las baterías.

7) El período formal de pruebas y regulaciones es de aproximadamente 14 días. Tomar datos. Ver figura $\mathrm{N} .{ }^{\circ} 5$.

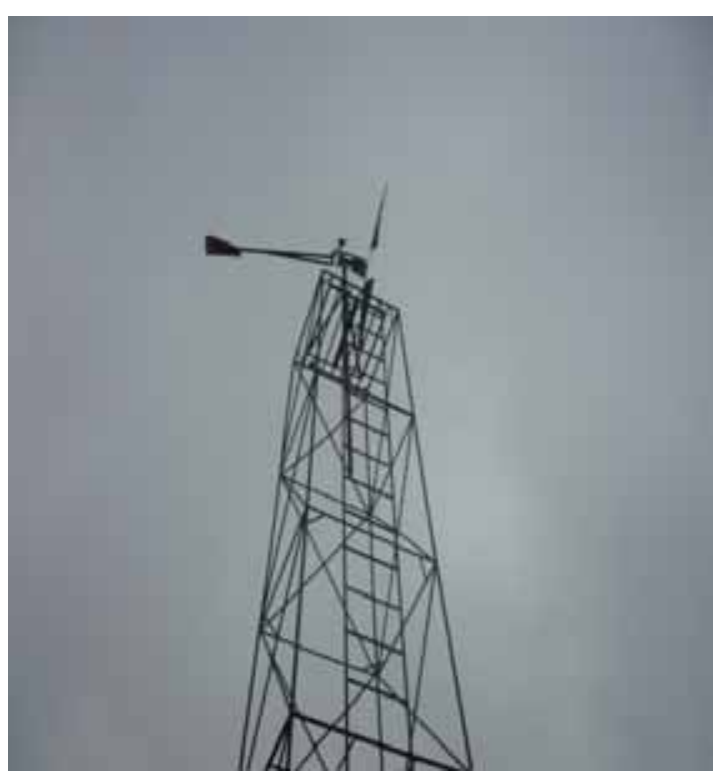

Figura $\mathbf{N}^{\circ}{ }^{5}$. Instalación del microaerogenerador en la EAP de Ingeniería de Minas de la UNMSM. 


\section{RESULTADOS DE LA CONSTRUCCIÓN DEL MICROAEROGENERADOR AR-SF-WP-500.}

Se obtuvo un aerogenerador con capacidad de proveer electricidad de 12 voltios en promedio, en realidad genera mayor que doce voltios, esto asegura que siempre se tendrá como mínimo 12 voltios para acumular en las baterías, con esto en un sistema híbrido hacemos mover el molino de bolas y martillo que ha sido diseñado en este proyecto.

Según la Figura N. ${ }^{\circ} 6$, el minimolino tiene dos accesos, uno de ellos es de molienda de martillo y el segundo de bolas, es impulsado por energía eólica en un $30 \%$ y un $70 \%$ con energía convencional.

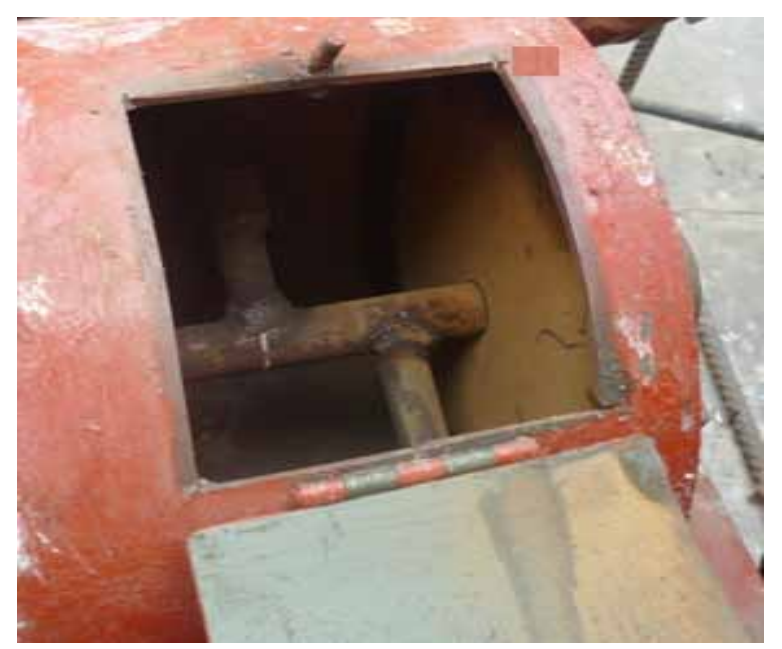

Figura $\mathbf{N}^{\circ}{ }^{6}$. Mini molino híbrido impulsado por el microaerogenerador AR-SF-WP-500.

\subsection{ANÁLISIS Y DISCUSIÓN DE RESULTADOS}

\subsubsection{Análisis de resultados}

La energía que se acumula del aerogenerador se acumula en las baterías convencionales, luego esta energía se lleva a 220 voltios mediante un conversor, a partir de la salida de 220 voltios se lleva a un motor de 0.88 caballos de potencia y con esto se hace mover el motor del molino híbrido hasta la obtención de los finos de malla 280 del relave a procesar, a partir de esto se somete a agitación con el mismo sistema de uso de la energía eólica y se culmina el ciclo de proceso de obtención de agregados de construcción mediante los mini equipos impulsados por energía eólica.

\subsubsection{Discusión de resultados}

La actual estructura de la matriz energética del Perú sugiere ser revisada. El desabastecimiento cíclico del mercado doméstico, el comportamiento inestable de los precios de los servicios eléctricos y de los combustibles y las crecientes presiones del crecimiento demográfico y el crecimiento demográfico y el crecimiento económico; sugieren que es poco sostenible en el mediano plazo, mantener la mezcla energética actual.

Se requiere un esfuerzo sostenido de la búsqueda de opciones energéticas consistentes con la necesidad de la seguridad energética del país, lo que incluye el desarrollo de la energía renovable y la energía nuclear.

\section{CONCLUSIONES}

Una vez conseguida la transformación de la energía cinética del viento en energía utilizable por el hombre, nos queda cómo conservarla, proceso en el cual hoy en día se están invirtiendo muchas horas de investigación para poder obtener el máximo aprovechamiento a posteriori, ya que normalmente el viento no es constante durante mucho tiempo. En consecuencia, tenemos que conservar la energía que obtenemos de él.

Es en ese sentido, que la Universidad Nacional Mayor de San Marcos a través de la Gestión del Vicerrectorado de Investigación (VRI) financia el Proyecto de Investigación Multidisciplinario "Mini equipos impulsados por energía eólica para obtener materiales de construcción a partir de relaves mineros", proyecto desarrollado en la Escuela de Ingeniería de Minas, que busca dar un aporte al conocimiento tecnológico en el rol de las energías no convencionales en el país.

En ese sentido, es muy importante que se planifique y se organicen Centros de Innovación Tecnológica Científica en este rubro, no solo en la energía eólica sino también en la energía solar, energía proveniente de hidrógeno y otros afines a favor del desarrollo del país.

\section{REFERENCIAS BIBLIOGRÁFICAS}

[1] Chiri Adolfo F. y Luyo Jaime E. La Seguridad Energética: Un Reto para el Perú en el Siglo XXI. Colegio de Ingenieros del Perú, 2008, Lima-Perú.

[2] Pacheco Werner, Romero Alfonso y Flores Silvana, Manual de Diseño y Construcción de un Microaerogenerador, Modelo AR-SF-WP-500, CONCYTEC, 2010. 